ISSN 0103-9954

\title{
CRESCIMENTO EM CAMPO DE ESPÉCIES FLORESTAIS EM DIFERENTES CONDIÇÕES DE ADUBAÇÕES
}

\author{
FIELD GROWTH OF FOREST SPECIES IN DIFFERENT CONDITIONS OF FERTILIZATIONS
}

\author{
Carlos Alberto Martinelli de Souza ${ }^{1}$ Rone Batista de Oliveira ${ }^{2}$ \\ Sebastião Martins Filho ${ }^{3}$ Julião Soares de Souza Lima ${ }^{4}$
}

\section{RESUMO}

O objetivo deste trabalho foi avaliar em condições de campo o comportamento de quatro espécies florestais submetidas a diferentes adubações orgânicas e minerais com adição de um condicionador de solo (Hidroplan). O delineamento estatístico adotado foi em blocos casualizados em parcelas subdivididas com trinta tratamentos que consistiram nas combinações dos fatores (6 adubos x 5 épocas) com cinco repetições, sendo usada uma planta por parcela. Foram avaliadas as características altura (h) e diâmetro das plantas (d). Os dados foram submetidos à análise de variância e ao teste de média (Newman-Keuls 5\%). As mudas foram produzidas em viveiro em tubetes de polietileno e plantadas em campo aos 90 dias após a rustificação. As adubações testadas no experimento foram: esterco bovino; esterco de galinha; adubação mineral NPK (testemunha); esterco de boi + Hidroplan; esterco de galinha + Hidroplan e adubação mineral NPK + Hidroplan. Os adubos orgânicos estudados contribuíram para melhor desempenho em todas as espécies, com exceção do Eucalyptus urophylla, que teve melhor desempenho no NPK e NPK + Hidroplan.

Palavras-chave: adubação química; adubação orgânica; altura; diâmetro.

\begin{abstract}
The work's objective was to evaluate, in field conditions, four forest species behavior submitted to different organic manurings and minerals with the addition of a conditioning of soil (Hidroplan). The adopted statistical outlining was in blocks, structured in portions subdivided with 30 treatments that consisted of combinations of factors (6 fertilizers x 5 times) with 5 repetitions, being used one plant by portion. Height (h) and diameter of the plants (d) were assessed. The data were submitted to the variance analysis and the average test (Newman-Keuls 5\%). The seedlings were produced in a nursery, in inside polyethylene tubas and they ware planted in field 90 days after the rustic. The manurings tested in the experiment were: bovine manure; chicken manure; mineral manuring NPK (testifies); bovine manure + Hidroplan; chicken manure + Hidroplan and mineral manuring NPK + Hidroplan. The studied organic fertilizers contributed to a better acting in every species, except for the Eucalyptus urophylla, which had a better acting in NPK and NPK + Hidroplan.
\end{abstract}

Keywords: mineral fertilization; organic fertilization; height; diameter.

\section{INTRODUÇÃO}

A produção de mudas de espécies florestais em larga escala para plantios comerciais, recuperação de áreas degradadas e recomposição de florestas faz com que haja grande procura por alternativas que visam à redução dos custos de manejo dessas espécies. Atualmente existem no mercado adubos comerciais que são usados para produção de mudas no viveiro e pós-plantio. Produzir mudas resistentes, mais capacitadas a sobreviver às adversidades encontradas no campo e uma boa adubação é uma das possíveis opções para minimizar as perdas pós-plantio.

Nesta última década o estudo de espécies florestais tornou-se uma realidade (Ventura e Rambelli, 1996). A necessidade de produção de mudas para plantios comerciais e recuperação de áreas degradadas tem promovido o desenvolvimento de tecnologias que envolvam a redução dos custos de manejo dessas mudas

1. Engenheiro Florestal, Mestrando em Produção Vegetal, Universidade Federal do Espírito Santo, Caixa Postal 16, CEP 29500-000, Alegre (ES). camartinelli@hotmail.com

2. Engenheiro Agrônomo, Mestrando em Produção Vegetal, Universidade Federal do Espírito Santo, Caixa Postal 16, CEP 29500-000, Alegre (ES).

3. Engenheiro Florestal, Dr., Professor Adjunto do Departamento de Estatística, Centro de Ciências Agrárias, Universidade Federal de Viçosa, CEP 36570-000, Viçosa (MG). smartins@cca.ufes.br

4. Engenheiro Agrícola, Dr., Professor Adjunto do Departamento de Engenharia Rural, Universidade Federal do Espírito Santo, Caixa Postal 16, CEP 29500-000, Alegre (ES). juliaosslima@cca.ufes.br

Recebido para publicação em 10/09/2004 e aceito em 24/07/2006. 
no viveiro e um bom desenvolvimento no campo (Coutinho e Carvalho, 1993).

Dentre outros fatores de natureza silvicultural, a nutrição das mudas via adubação de seus substratos de crescimento desponta como um dos principais responsáveis pela obtenção de sua maior produtividade (nos viveiros) e qualidade, além da maior economicidade do processo de sua produção (Neves et al., 1990).

Os estercos animais vêm sendo empregados como fertilizantes a mais de dois mil anos (Kiehl, 1985). Os adubos orgânicos, além do fornecimento de nutrientes, destacam-se por um papel fundamental e mais importante, fornecimento de matéria orgânica para melhorar as propriedades físicas, químicas e biológicas do solo. Nesse caso, o efeito é o condicionador de solo, considerando a matéria orgânica como um produto químico que melhora as propriedades físicas do solo (Rajj, 1991).

Segundo Malavolta et al., (2004), alguns adubos orgânicos são empregados em doses elevadas, toneladas por hectares, e são pobres nos elementos nutritivos como nitrogênio, fósforo e potássio. Valem especialmente pela matéria orgânica que, incorporada ao solo, se decompõe e forma húmus. Outros, mais concentrados, comportam-se de modo mais semelhante ao dos adubos minerais, funcionando como fonte de nitrogênio, fósforo, potássio e outros elementos.

Em análises realizadas com esterco bovino indicaram que, em uma tonelada, contém 5 quilos de nitrogênio, 2,5 quilos de $\mathrm{P}_{2} \mathrm{O}_{5}$ e 5 quilos de $\mathrm{K}_{2} \mathrm{O}$. As mesmas análises mostram que quase a metade do nitrogênio do esterco vem da urina enquanto que no caso do potássio mais da metade estava nela, quanto ao fósforo praticamente todo ele vem das fezes (Malavolta, 1989).

Embora alguns estudos acerca dos requerimentos nutricionais e respostas ao uso de nutrientes em espécies florestais já tenham sido conduzidos, a maioria deles foram realizados em casa de vegetação (Dias e Faria, 1992). Mesmo gerando resultados importantes sobre as exigências nutricionais e respostas das espécies à fertilização, estes têm aplicação restrita para recomendação da adubação no campo pelo fato de poucos estudos terem sido realizados nessas condições.

O objetivo deste trabalho foi avaliar em condições de campo, o comportamento de quatro espécies florestais submetidas a diferentes adubações orgânicas e minerais com adição de um condicionador de solo (Hidroplan) com o intuito de reter água.

\section{MATERIAL E MÉTODOS}

O trabalho foi conduzido durante o período de fevereiro a junho de 2003 na estação experimental do Centro de Ciências Agrárias da Universidade Federal do Espírito Santo, no município de Alegre, ES, situado a $20^{\circ} 45^{\prime} 48^{\prime \prime}$ de latitude Sul e 41 $31^{\prime} 57^{\prime \prime}$ de longitude Oeste de Greenwich, altitude de aproximadamente 150 metros. O clima predominante é quente e úmido no verão com inverno seco, com uma precipitação anual média de $1200 \mathrm{~mm}$ e temperatura média anual de $23^{\circ} \mathrm{C}$, com máximas diárias de $29^{\circ} \mathrm{C}$ e mínimas de $20^{\circ} \mathrm{C}$ (Espírito Santo, 1994).

As sementes utilizadas foram coletadas pelos alunos bolsistas do laboratório de sementes da Universidade, as plantas matrizes são da própria região onde o experimento foi conduzido. As mudas foram produzidas em viveiro em tubetes de polietileno de secção circular com diâmetro superior de $2,5 \mathrm{~cm}$ e altura de $12 \mathrm{~cm}$ com capacidade volumétrica de $50 \mathrm{~cm}^{3}$. Foram distribuídas duas sementes por tubetes e, após 15 dias, efetuou-se a seleção, mantendo-se a plântula mais vigorosa. As espécies produzidas foram: Eucalyptus urophylla (eucalipto), Cedrela odorata (cedro-rosa), Schinnus terebinthifolius (aroeira) e Acacia holosericea (acácia). Essas espécies foram escolhidas por apresentarem crescimento rápido e outras potencialidades econômicas.

Os substratos utilizados foram compostos à base de 30\% de casca de amendoim processada, $30 \%$ de húmus de minhoca, $17 \%$ de areia, $3 \%$ de terra de barranco, fertilizante e calcário. Amostras do substrato foram analisadas, resultando com valores de $0,63\left(\mathrm{~g} \cdot \mathrm{cm}^{3}\right), 63 \%, 192(\mathrm{mg} / \mathrm{dm}), 880(\mathrm{mg} / \mathrm{dm}), 9,4\left(\mathrm{cmol}_{\mathrm{c}} \mathrm{dm}^{-3}\right)$, 2,8 $\left(\mathrm{cmol}_{\mathrm{c}} \mathrm{dm}^{-3}\right), 16,7\left(\mathrm{cmol}_{c} \mathrm{dm}^{-3}\right)$ e 320 para a densidade do solo, porosidade total, $\mathrm{P}, \mathrm{K}$ trocável, Ca trocável $\mathrm{Mg}$ trocável, $\mathrm{Mg}$ trocável $\left(\mathrm{cmol}_{\mathrm{c}} \mathrm{dm}^{-3}\right)$ e matéria orgânica total.

Depois do término de produção que se deu 60 dias após a germinação, as mudas passaram por um processo de rustificação que durou mais 30 dias para depois ser feito o plantio em campo. O solo da área em que foi efetuado o plantio em campo é classificado como Latossolo Vermelho Distrófico (EMBRAPA, 
1999), textura média, com $311,13 \mathrm{~g} \mathrm{~kg}^{-1}$ de argila, $330,96 \mathrm{~g} \mathrm{~kg}^{-1}$ de silte e $357,91 \mathrm{~g} \mathrm{~kg}^{-1}$ de areia, proveniente da camada de 0 a $20 \mathrm{~cm}$ de profundidade. As características químicas do solo são: $\mathrm{pH}\left(\mathrm{H}_{2} \mathrm{O}\right)=5,6 ; \mathrm{Ca}^{2+}=3,8$ $\mathrm{cmol}_{\mathrm{c}} \mathrm{dm}^{-3} ; \mathrm{Mg}^{2+}=1,8 \mathrm{cmol}_{\mathrm{c}} \mathrm{dm}^{-3} ; \mathrm{P}=42 \mathrm{mg} \mathrm{dm}{ }^{-3} ; \mathrm{K}=114 \mathrm{mg} \mathrm{dm} \mathrm{dm}^{-3} \mathrm{e}+\mathrm{Al}=2,5 \mathrm{cmol}_{\mathrm{c}} \mathrm{dm}^{-3}$, determinadas segundo EMBRAPA (1997). Os tratos culturais realizados na área do plantio foram uma simples roçagem mecânica e controle de formigas cortadeiras.

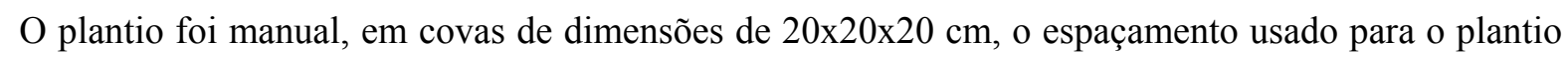
foi de 1,5x1,5 m. Para o fator adubação foram testadas no experimento: 1 - esterco de boi ( 2 litros/cova); 2 esterco de galinha (1 litro/cova); 3 - adubação mineral (testemunha) NPK20-05-15 (75g/cova); 4 - esterco de boi (2litros/cova) + Hidroplan (7 g/cova); 5 - esterco de galinha (1litro/cova) + Hidroplan (7 g/cova) e 6 adubação mineral (testemunha) NPK- 20-05-15 + Hidroplan (7 g/cova). O Hidroplan é um produto comercial hidroabsorvente que tem como objetivo reter e deixar água disponível ao sistema radicular das plantas.

O delineamento estatístico adotado foi em blocos casualizados em parcelas subdivididas com trinta tratamentos (6 adubos x 5 épocas) com cinco repetições, sendo usada uma planta por parcela. A cada 30 dias, foram avaliadas as seguintes características: altura da planta e diâmetro do coleto. Após a coleta dos dados, foram realizadas análises estatísticas, e quando houve diferença significativa, estudoaram-se o fator adubo pelo teste de Newman-Keuls $(\mathrm{p}<0,05)$ e o fator época pela análise de regressão.

\section{RESULTADOS E DISCUSSÃO}

Os dados apresentados na Tabela 1 mostram os resultados dos quadrados médios para as interações. Verifica-se que a interação época $\mathrm{x}$ adubo foi significativa pelo teste $\mathrm{F}$ para quase-todos os parâmetros com exceção apenas para altura e diâmetro de Acacia holosericea e da altura para Eucalyptus urophylla, portanto para avaliar essas características morfológicas nessas espécies essa interação não representa um obstáculo, pois em qualquer época após o plantio, as plantas já estão em condições de serem avaliadas, o que não ocorre nos outros casos que apresentaram interatividade, e os fatores devem ser estudados levando-se em consideração os adubos e a respectiva época de análise.

TABELA 1: Quadrados médios da altura de plantas $-\mathrm{h}(\mathrm{cm})$, e diâmetro do coleto $-\mathrm{d}(\mathrm{mm})$, de quatro espécies de espécies florestais. Alegre, ES, 2003.

TABLE 1: Medium squares of the height of plants $-\mathrm{h}(\mathrm{cm})$, and diameter of the stem $-\mathrm{d}(\mathrm{mm})$, of four species of forest species. Alegre, ES, 2003.

\begin{tabular}{|c|c|c|c|c|c|c|c|c|}
\hline \multirow[t]{2}{*}{ FV } & \multicolumn{2}{|c|}{ Schinus terebinthifolius } & \multicolumn{2}{|c|}{ Acacia holosericea } & \multicolumn{2}{|c|}{ Eucalyptus urophylla } & \multicolumn{2}{|c|}{ Cedrela odorata $L$. } \\
\hline & $\mathrm{h}(\mathrm{cm})$ & $\mathrm{d}(\mathrm{cm})$ & $\mathrm{h}(\mathrm{cm})$ & $\mathrm{d}(\mathrm{cm})$ & $\mathrm{h}(\mathrm{cm})$ & $\mathrm{d}(\mathrm{cm})$ & $\mathrm{h}(\mathrm{cm})$ & $\mathrm{d}(\mathrm{cm})$ \\
\hline Bloco & 162,1 & 2,3 & 537,0 & 9,7 & 209,3 & 18,0 & 24,5 & 1,7 \\
\hline Adubo & $508,1 *$ & $10,4 *$ & $939,9 *$ & $7,8^{\mathrm{ns}}$ & $476,4^{\mathrm{ns}}$ & $33,8^{\mathrm{ns}}$ & $176,9^{\text {ns }}$ & $30,4 *$ \\
\hline Resíduo & 89,1 & 2,9 & 257,7 & 3,3 & 508,1 & 7,9 & 85,6 & 2,5 \\
\hline Época & $2711,5 *$ & $160,2 *$ & $33680,6 *$ & $396,1 *$ & $8689,4 *$ & $351,7 *$ & $5547,2 *$ & 224,9 \\
\hline Época adubo* & $43,0 *$ & $1,9 *$ & $67,8^{\mathrm{ns}}$ & $1,2^{\mathrm{ns}}$ & $215,0^{\mathrm{ns}}$ & $5,9 *$ & $23,6 *$ & $3,1 *$ \\
\hline Resíduo & 17,1 & 0,7 & 55,5 & 1,1 & 156,5 & 2,0 & 4,1 & 0,3 \\
\hline Média & 32 & 5,81 & 70,3 & 7,7 & & 6,7 & 30,9 & 6,4 \\
\hline$\overline{\mathrm{CV}(\%)}$ & 12,6 & 14,1 & 10,6 & 13,2 & 24,6 & 21,4 & 6,6 & 8,3 \\
\hline
\end{tabular}

Em que: * = significativo ao nível de $5 \%$ de probabilidade; ns = não-significativo ao nível de $5 \%$ de probabilidade.

A utilização de espécies nativas, em plantios com fins comerciais torna-se comprometida pelo pouco conhecimento do comportamento silvicultural dessas espécies sobretudo em relação às suas exigências nutricionais. Neste trabalho, resultados apresentados na Tabela 2 mostram as médias dos parâmetros morfológicos avaliados nas plantas de Schinus terebinthifolius (aroeira). Não existe significância estatística nas duas primeiras análises e partindo da terceira coleta de dados às diferenças entre o crescimento das plantas nos respectivos adubos começaram a ficar evidentes. Em relação à altura, aos 120 dias, os adubos 2, 4 e 5 foram os que apresentaram melhor desempenho assim como os adubos 1,2 e 4 foram os melhores para incremento no diâmetro do coleto.

É importante destacar que o diâmetro do coleto é de fundamental importância na avaliação do potencial da muda para sobrevivência e crescimento após o plantio. Segundo Carneiro (1983), as plantas com maior diâmetro apresentam maior sobrevivência, especialmente pela maior capacidade de formação e de 
crescimento de novas raízes.

TABELA 2: Altura - h $(\mathrm{cm})$ e diâmetro do coleto $-\mathrm{d}(\mathrm{mm})$, de Schinnus terebinthifolius plantada com diferentes adubos em diferentes épocas. Alegre, ES, 2003.

TABLE 2: Schinnus terebinthefolius height $-\mathrm{h}(\mathrm{cm})$ and diameter of the stem $-\mathrm{d}(\mathrm{mm})$ planted with different fertilizers in different times. Alegre, ES, 2003.

\begin{tabular}{|c|c|c|c|c|c|c|c|c|c|c|}
\hline \multirow[t]{2}{*}{ Adubos } & \multicolumn{2}{|c|}{ Época 0} & \multicolumn{2}{|c|}{ Época 30} & \multicolumn{2}{|c|}{ Época 60} & \multicolumn{2}{|c|}{ Época 90} & \multicolumn{2}{|c|}{ Época 120} \\
\hline & $\mathrm{h}(\mathrm{m})$ & $\mathrm{d}(\mathrm{cm})$ & $h(m)$ & $\mathrm{d}(\mathrm{cm})$ & $h(m)$ & $\mathrm{d}(\mathrm{cm})$ & $\mathrm{h}(\mathrm{m})$ & $\mathrm{d}(\mathrm{cm})$ & $\mathrm{h}(\mathrm{m})$ & $\mathrm{d}(\mathrm{cm})$ \\
\hline 1 & $16,6 \mathrm{a}^{1}$ & $2,7 \mathrm{a}$ & $22,4 \mathrm{a}$ & $3,6 \mathrm{a}$ & $25,4 \mathrm{a}$ & $5,6 \mathrm{ab}$ & $34,8 \mathrm{~cd}$ & $7,8 \mathrm{a}$ & $38,60 \mathrm{~b}$ & $9,40 \mathrm{a}$ \\
\hline 2 & $20,4 \mathrm{a}$ & $3,4 \mathrm{a}$ & $32,6 \mathrm{a}$ & $4,8 \mathrm{a}$ & $40,0 \mathrm{a}$ & $6,4 \mathrm{ab}$ & $44,2 \mathrm{ab}$ & $8,0 \mathrm{a}$ & $50,20 \mathrm{a}$ & $9,40 \mathrm{abc}$ \\
\hline 3 & $20,0 \mathrm{a}$ & $3,2 \mathrm{a}$ & $26,6 \mathrm{a}$ & $4,4 \mathrm{a}$ & $27,8 \mathrm{a}$ & $5,2 \mathrm{a}$ & $28,8 \mathrm{~cd}$ & $5,6 \mathrm{~b}$ & $34,80 \mathrm{~b}$ & $6,80 \mathrm{~d}$ \\
\hline 4 & $19,8 \mathrm{a}$ & $3,2 \mathrm{a}$ & $29,6 \mathrm{a}$ & $4,8 \mathrm{a}$ & $40,4 \mathrm{a}$ & $6,8 \mathrm{a}$ & $46,4 \mathrm{a}$ & $8,4 \mathrm{a}$ & $49,20 \mathrm{a}$ & $10,20 \mathrm{a}$ \\
\hline 5 & $22,0 \mathrm{a}$ & $2,8 \mathrm{a}$ & $29,4 \mathrm{a}$ & $3,8 \mathrm{a}$ & $35,2 \mathrm{a}$ & $5,4 \mathrm{~b}$ & $41,8 \mathrm{abc}$ & $8,0 \mathrm{a}$ & $47,20 \mathrm{a}$ & $8,60 \mathrm{~b}$ \\
\hline 6 & $17,7 \mathrm{a}$ & $2,6 \mathrm{a}$ & $282 \mathrm{a}$ & $4,0 \mathrm{a}$ & $32,2 \mathrm{a}$ & $5,0 \mathrm{~b}$ & $38,6 \mathrm{bc}$ & $6,6 \mathrm{ab}$ & $43,20 \mathrm{~b}$ & $7,80 \mathrm{c}$ \\
\hline
\end{tabular}

Médias seguidas pela mesma letra não diferem significativamente, pelo teste de Newman Keuls ao nível de $5 \%$ de probabilidade.

Na Tabela 3, observam-se as médias das alturas e dos diâmetros das plantas de Cedrela odorata. Nessa espécie o crescimento em diâmetro acorreu com maior intensidade se comparado com a altura, na $3^{\text {a }}$ avaliação, existe diferença significativa entre as médias, sendo que os tratamentos 1,2 e 5 apresentaram melhores desempenhos.

O crescimento da parte aérea foi mais lento em relação ao diâmetro, sendo que só houve constatação de significância entre as médias após a $4^{\mathrm{a}}$ avaliação, e os tratamentos $1,2,3$ e 5 obtiveram melhores respostas nesta característica. Nota-se que, para essa espécie assim como para altura em Schinnus terebinthifolius e Acacia holosericea, os adubos à base de esterco de galinha sempre condicionaram os maiores crescimentos.

O crescimento em altura nessa espécie pode ter sido afetado pelo fato de se tratar de uma planta secundária, que exige melhores condições de sombreamento para ter melhor desenvolvimento, ou seja, tem melhor desempenho em povoamentos já desenvolvidos. Segundo Scalon et al., (2002), a capacidade de crescer rapidamente, quando sombreada, é um mecanismo importante de adaptação das espécies, o que constitui uma valiosa estratégia para escapar das condições de baixas intensidades luminosas.

TABELA 3: Altura de plantas $-\mathrm{h}(\mathrm{cm})$ e diâmetro do coleto $-\mathrm{d}(\mathrm{mm})$, de Cedrela odorata plantada com diferentes adubos em diferentes épocas, Alegre, ES, 2003.

TABLE 3: Cedrela odorata's height $-\mathrm{h}(\mathrm{cm})$ and diameter of the stem $-\mathrm{d}(\mathrm{mm})$ planted with different fertilizers in different times, Alegre, ES, 2003.

\begin{tabular}{|c|c|c|c|c|c|c|c|c|c|c|}
\hline \multirow[t]{2}{*}{ Adubos } & \multicolumn{2}{|c|}{ Época 0} & \multicolumn{2}{|c|}{ Época 30} & \multicolumn{2}{|c|}{ Época 60} & \multicolumn{2}{|c|}{ Época 90} & \multicolumn{2}{|c|}{ Época 120} \\
\hline & $\mathrm{h}(\mathrm{m})$ & $\mathrm{d}(\mathrm{cm})$ & $\mathrm{h}(\mathrm{m})$ & $\mathrm{d}(\mathrm{cm})$ & $\mathrm{h}(\mathrm{m})$ & $\mathrm{d}(\mathrm{cm})$ & $\mathrm{h}(\mathrm{m})$ & $\mathrm{d}(\mathrm{cm})$ & $\mathrm{h}(\mathrm{m})$ & $\mathrm{d}(\mathrm{cm})$ \\
\hline 1 & $13,2 \mathrm{a}^{1}$ & $3,4 \mathrm{a}$ & $13,5 \mathrm{a}$ & $5,3 \mathrm{a}$ & $23,5 \mathrm{a}$ & $7,3 \mathrm{a}$ & $44,8 \mathrm{a}$ & $10,0 \mathrm{a}$ & $46,00 \mathrm{abc}$ & $12,00 \mathrm{a}$ \\
\hline 2 & $15,6 \mathrm{a}$ & $3,8 \mathrm{a}$ & $21,6 \mathrm{a}$ & $4,8 \mathrm{a}$ & $32,6 \mathrm{a}$ & $7,4 \mathrm{a}$ & $42,4 \mathrm{ab}$ & $9,0 \mathrm{ab}$ & $51,20 \mathrm{ab}$ & $11,20 \mathrm{a}$ \\
\hline 3 & $14,8 \mathrm{a}$ & $3,0 \mathrm{a}$ & $20,2 \mathrm{a}$ & $5,0 \mathrm{a}$ & $30,4 \mathrm{a}$ & $6,8 \mathrm{a}$ & $42,0 \mathrm{ab}$ & $8,0 \mathrm{~b}$ & $50,40 \mathrm{ab}$ & $10,00 \mathrm{~b}$ \\
\hline 4 & $14,3 \mathrm{a}$ & $3,3 \mathrm{a}$ & $19,8 \mathrm{a}$ & $3,5 \mathrm{a}$ & $26,0 \mathrm{a}$ & $4,8 \mathrm{~b}$ & $34,0 \mathrm{bc}$ & $5,5 \mathrm{c}$ & $42,50 \mathrm{c}$ & $7,25 \mathrm{~d}$ \\
\hline 5 & $15,4 \mathrm{a}$ & $3,2 \mathrm{a}$ & $23,5 \mathrm{a}$ & $4,8 \mathrm{a}$ & $33,0 \mathrm{a}$ & $6,0 \mathrm{a}$ & $43,7 \mathrm{a}$ & $8,8 \mathrm{ab}$ & $53,75 \mathrm{a}$ & $11,75 \mathrm{a}$ \\
\hline 6 & $13,4 \mathrm{a}$ & $3,0 \mathrm{a}$ & $20,5 \mathrm{a}$ & $3,5 \mathrm{a}$ & $26,5 \mathrm{a}$ & $4,8 \mathrm{~b}$ & $35,5 \mathrm{bc}$ & $6,3 \mathrm{c}$ & $44,00 \mathrm{bc}$ & $8,75 \mathrm{c}$ \\
\hline
\end{tabular}

Médias seguidas pela mesma letra não diferem significativamente, pelo teste de Newman Keuls ao nível de $5 \%$ de probabilidade.

As plantas de Eucalyptus urophylla diferiram significativamente, com as médias apresentando distinção no desenvolvimento somente para o diâmetro do coleto partindo da $3^{\mathrm{a}}$ análise, e aos 120 e dias os adubos 1,3 e 6 foram os que tiveram melhores respostas, como mostrado na Tabela 4 . O que mostra que, à exceção da altura para a espécie Cedrela odorata, as adubações minerais testadas proporcionaram desempenho superior para essa variável nessa espécie.

De acordo com Costa Filho (1992), a adubação mineral tem sido sistematicamente utilizada no Brasil com bons resultados, para espécies dos gêneros Pinus e Eucalyptus. Contudo, sua utilização para essências 
florestais nativas tem sido menos freqüente.

TABELA 4: Diâmetro do coleto - d (mm), de plantas de Eucalyptus urophylla plantado com diferentes adubos em diferentes épocas, Alegre, ES, 2003.

TABLE 4: Eucalyptus urophylla's diameter of stem $-\mathrm{d}(\mathrm{mm})$ plants of planted with different fertilizers in different times, Alegre, ES, 2003.

\begin{tabular}{cccccccc}
\hline Adubos & Época 0 & Época 30 & Época 60 & Época 90 & Época 120 \\
\hline 1 & $2,6 \mathrm{a}^{1}$ & $4,0 \mathrm{a}$ & $8,0 \mathrm{a}$ & $9,6 \mathrm{a}$ & $14,4 \mathrm{a}$ \\
2 & $2,2 \mathrm{a}$ & $4,6 \mathrm{a}$ & $7,0 \mathrm{a}$ & $8,4 \mathrm{a}$ & $9,6 \mathrm{~cd}$ \\
3 & $2,0 \mathrm{a}$ & $5,8 \mathrm{a}$ & $7,8 \mathrm{a}$ & $9,4 \mathrm{a}$ & $12,6 \mathrm{ab}$ \\
4 & $2,0 \mathrm{a}$ & $3,2 \mathrm{a}$ & $4,6 \mathrm{~b}$ & $5,8 \mathrm{~b}$ & $7,4 \mathrm{~d}$ \\
5 & $2,8 \mathrm{a}$ & $4,8 \mathrm{a}$ & $7,0 \mathrm{ab}$ & $8,6 \mathrm{a}$ & $10,2 \mathrm{bc}$ \\
6 & $2,2 \mathrm{a}$ & $4,4 \mathrm{a}$ & $7,4 \mathrm{a}$ & $10,8 \mathrm{a}$ & $14,6 \mathrm{a}$ \\
\hline
\end{tabular}

Médias seguidas pela mesma letra não diferem significamente, pelo teste de Newman Keuls ao nível de 5\% de probabilidade.

A Tabela 5 mostra as médias da altura e diâmetro do coleto para Acacia holosericea e altura para Eucalyptus urophylla. Esses dados são referentes à última análise, que ocorreu 120 dias após plantio, como para essas características não houve interatividade entre épocas de coleta de dados e adubos, esses fatores puderam ser estudados de forma independente pelo teste de médias Em relação ao parâmetro altura em Acacia holosericea, verifica-se que os adubos 1, 2 e 5 foram os que apresentaram melhores resultados. Essa espécie foi a que dentre todas teve melhor crescimento em altura para todos os adubos. Conforme Barbosa et al., (1992), os resultados para certas espécies podem ser reflexo do comportamento próprio de cada essência, sem contudo estarem associados aos conceitos teóricos de grupos sucessionais, os quais não foram ainda confirmados para algumas espécies. Silva et al., (1997), avaliando a resposta ao potássio, envolvendo várias espécies florestais, relacionaram o seu comportamento diante dos tratamentos à sua classificação de acordo com o grupo sucessional. Esses autores sugerem que a elevada resposta de algumas espécies parece ser por causa da sua maior taxa de crescimento, requerendo, desse modo, uma maior quantidade de nutrientes para atender à sua demanda nutricional, o que, em última análise, permite a expressão do potencial de produção de biomassa das espécies de crescimento inicial mais acentuado. Apesar dessa consideração, os resultados obtidos no presente trabalho indicam que as espécies mais responsivas foram aquelas com as maiores taxas de crescimento, independentemente do seu grupo sucessional.

TABELA 5: Altura - h (cm) e diâmetro do coleto - d (mm) de Acacia holocericea e altura de Eucalyptus urophylla, plantadas em diferentes adubos em diferentes épocas. Alegre, ES, 2003.

TABLE 5: Acacia holocericea's height $-\mathrm{h}(\mathrm{cm})$ and diameter of the stem $-\mathrm{d}(\mathrm{mm})$, and Eucalyptus urophylla's height planted in different fertilizers in different times. Alegre, ES, 2003.

\begin{tabular}{c|cc|c}
\hline \multirow{2}{*}{ Adubos } & \multicolumn{2}{|c|}{ Acacia holosericea } & Eucalyptus urophylla \\
\cline { 2 - 4 } & $\mathrm{h}(\mathrm{m})$ & $\mathrm{d}(\mathrm{cm})$ & $\mathrm{h}(\mathrm{m})$ \\
\hline 1 & $73,5 \mathrm{ab}^{1}$ & $8,2^{2}$ & $50,4^{2}$ \\
2 & $74,6 \mathrm{a}$ & 8,2 & 48,5 \\
3 & $63,5 \mathrm{c}$ & 7,3 & 54,6 \\
4 & $62,8 \mathrm{c}$ & 6,9 & 44,0 \\
5 & $77,7 \mathrm{a}$ & 8,2 & 56,3 \\
6 & $69,8 \mathrm{~b}$ & 8,2 & 51,0 \\
\hline
\end{tabular}

Em que: 1 = as médias seguidas pela mesma letra não diferem significativamente, pelo teste de Newman Keuls ao nível de $5 \%$ de probabilidade; 2 = não-significativo ao nível de $5 \%$ de probabilidade pelo teste $\mathrm{F}$.

Para o crescimento em diâmetro da Acacia holosericea e altura do Eucalyptus urophylla não houve diferença significativa entre os adubos, porém todos apresentaram índice de crescimento satisfatório em comparação com plantas da mesma espécie e idade, submetidas a outros tipos de tratos silviculturais já consolidados.

Segundo Pritchett (1979), citado por Schumacher et al., (2003), a absorção dos nutrientes pelas árvores é influenciada pela espécie, pela cobertura florestal e pelas condições de solo e clima. Em princípio, 
a absorção anual de nutrientes pela maioria das espécies florestais é da mesma ordem da apresentada pelas culturas agrícolas, mas como a maior parte dos nutrientes absorvidos é devolvida para o piso florestal, quantidades relativamente pequenas são retidas no acréscimo anual da biomassa arbórea. Sendo assim, a diferença entre as diferentes adubações está na quantidade e na forma com que estas disponibilizam os nutrientes para as plantas.

O fato de este experimento ter sido implantado no período chuvoso, talvez não tenha possibilitado o hidroplan de expressar a sua potencialidade visto que alguns tratamentos sem esse produto apresentaram desempenho igual ou melhor. Resultados positivos foram obtidos com o hidrogel Stockosorb 500 em relação à sobrevivência e crescimento de mudas de Eucalyptus urophylla plantadas na época seca no município de Piracicaba, SP e com o Sotcksorb K 400 com Pinus halepensis sob situação de estresse hídrico em casa de vegetação (Buzetto et al. 2001).

\section{CONCLUSÕES}

Para este estudo, pode-se concluir que os adubos orgânicos estudados tiveram melhores desempenhos em todas espécies, com destaque para o esterco de galinha, com exceção do Eucalyptus urophylla, que se desenvolveu melhor quando submetido a adubação com NPK e NPK + hidroplan. Isso mostra que a adubação orgânica para uso no plantio dessas espécies é plenamente viável, podendo ser limitada apenas pela quantidade demandada em plantios em larga escala pelo fato de muitas propriedades não produzirem quantidades suficientes.

O hidroplan não apresentou o resultado esperado visto que alguns adubos sem esse produto apresentaram desempenho igual ou melhor.

Maior quantidade de pesquisas referentes a esse assunto deve ser realizada pelo fato do Brasil apresentar uma infinidade de espécies nativas e exóticas com pouco conhecimento sobre as diferentes respostas às práticas silviculturais por essas espécies, além do fato dos adubos orgânicos apresentarem diferenças dependendo da região onde é produzido por causa da variação na alimentação animal.

\section{REFERÊNCIAS BIBLIOGRÁFICAS}

BARBOSA, L.M.; ASPERTI, L.M.; BEDINELLI, C. Estudos sobre o estabelecimento e desenvolvimento de espécies com ampla ocorrência em mata ciliar. Revista do Instituto Florestal, São Paulo, v. 4, p. 605-608, 1992.

BUZETTO, F.A. Relatório Degussa Brasil Ltda. 2001. Disponível em: <www.degussa.com.br> Acesso em: 10 jan.2006.

CARNEIRO, J. G. A. Influência dos fatores ambientais e das técnicas de produção sobre o desenvolvimento de mudas florestais e a importância dos parâmetros que definem sua qualidade. In: SIMPÓSIO SOBRE FLORESTAS PLANTADAS NOS NEOTRÓPICOS COMO FONTE DE ENERGIA, 1983, Viçosa. Anais... Viçosa: Universidade Federal de Viçosa, 1983. p.10-24.

COSTA FILHO, R.T. da. Crescimento de mudas de aroeira (Astronium urundeuva Fr. All. Engl.) em resposta à calagem, fósforo e potássio. In: CONGRESSO NACIONAL SOBRE ESSÊNCIAS NATIVAS, 2., 1992 , São Paulo. Anais... São Paulo, 1992. v. 4, p. 537-543.

COUTINHO, C. J.; CARVALHO, C. M. O uso da vermiculita na produção de mudas florestais. In: ENCONTRO NACIONAL DE REFLORESTADORES, 7., 1983, Curitiba. Anais... 1983. p. 54-63.

DIAS, L. E.; FARIA, S. M.; FRANCO, A. A. Crescimento de mudas de Acacia mangiuns Wild, em resposta à omissão de macronutrientres. Revista Árvore, v. 18, n. 2, p. 123-131, 1994.

ESPÍRITO SANTO. Secretaria de Estado de Ações Estratégicas e Planejamento. Informações municipais do Estado do Espírito Santo, 1994. Vitória: Departamento Estadual de Estatística, 1994. v. 1. 803p.

KIEHL, E.J. Fertilizantes orgânicos. São Paulo : Pioneira, 1985. 492p.

MALAVOLTA, E. Abc da adubação. São Paulo : Ceres, 1989. 292p.

MAlAVOlTA, E.; GOMES,P,F.; ALCARDE, J.C. Adubos e adubações. São Paulo: Nobel, 2004. 1970p.

NETO, S. P. M.; GONÇALVES, J. L. M. Efeitos da luminosidade sobre o estado nutricional de mudas de espécies arbóreas que ocorrem na mata atlântica. Revista Árvore, Viçosa, v.25, n.1, p.28-38, 2001.

NEVES, J.C.L.; GOMES, J.M.; NOVAIS R. F.; BARROS, N. F. Fertilização mineral de mudas de eucalipto. In: 
Barros, N.F. ; Novais, R.F., eds. Relação solo-eucalipto. Viçosa: Folha de Viçosa, 1990. p. 99-126.

RAJJ, B, V. Fertilidade do solo e adubação. São Paulo: Ceres, 1991. 343p.

REIS, G. G.; REIS, M. G. F.; RODRIGUES, F. L.; BERNARDO, A. L.; GARCIA, N. F. P. Efeitos da poda de raízes de mudas de eucalipto produzidas em tubetes sobre a arquitetura do sistema radicular e o crescimento no campo. Revista Árvore, Viçosa, v. 20, n. 2, p. 137-146,1996.

SCALON, S. P. Q.; MUSSURI. M, R.; RIGONI. M. V. F. Crescimento inicial de mudas de espécies florestais nativas sob diferentes níveis de sombreamento. Revista Árvore, Viçosa, v. 26, n. 1, p. 1-5, 2002.

SCHUMACHER, M.V.; BRUN, E. J.; RODRIGUES.; L. M.; SANTOS, E. M. Retorno de nutrientes via deposição de serrapilheira em um povoamento de acácia-negra (Acacia mearnsii De Wild.) no Estado do Rio Grande do Sul. Revista Árvore, Viçosa, v. 27, n. 6, p. 791-798, 2003.

SILVA, I.R.; FURTINI NETO, A.E.; CURI, N.; VALE, F.R. Crescimento inicial de quatorze espécies florestais nativas em resposta à adubação potássica. Pesquisa Agropecuária Brasileira, Brasília, v.32, n.2, p.205-212, fev., 1997.

SOUZA, L. D. N. Adubação orgânica. Rio de Janeiro: Ediouro do campo, 1999. 115p.

VENTURA, V. J.; RAMBELLI, A. M. Legislação federal sobre o meio ambiente: leis, decreto-leis, decretos, portarias e resoluções anotados para uso prático e imediato. 2. ed. Taubaté: Vana, 1996. 1148p. 